\title{
Disturbance Attenuation via Output Feedback for Nonlinear Time-Delay Systems with Input Matching Uncertainty
}

\author{
Chao Guo ${ }^{1}$ and Kemei Zhang $\mathbb{D}^{2}$ \\ ${ }^{1}$ Institute of Automation, Qufu Normal University, Shandong Province 273165, China \\ ${ }^{2}$ School of Mathematics Sciences, Qufu Normal University, Shandong Province 273165, China \\ Correspondence should be addressed to Kemei Zhang; zhkm90@126.com
}

Received 14 April 2018; Accepted 13 June 2018; Published 5 July 2018

Academic Editor: Weihai Zhang

Copyright (c) 2018 Chao Guo and Kemei Zhang. This is an open access article distributed under the Creative Commons Attribution License, which permits unrestricted use, distribution, and reproduction in any medium, provided the original work is properly cited.

This paper studies the problem of output feedback disturbance attenuation for a class of uncertain nonlinear systems with input matching uncertainty and unknown multiple time-varying delays, whose nonlinearities are bounded by unmeasured states multiplying unknown polynomial-of-output growth rate. By skillfully combining extended state observer, dynamic gain technique, and Lyapunov-Krasovskii theorem, a delay-independent output feedback controller can be developed with only one dynamic gain to guarantee the boundedness of closed-loop system states and the achievement of global disturbance attenuation in the $L_{2}$-gain sense.

\section{Introduction}

The problem of disturbance attenuation via output feedback for nonlinear systems is a relatively meaningful problem in control theory and applications. Compared with the stabilization control and tracking control, fewer results on output feedback disturbance attenuation design have been obtained until now, such as [1-5] and the references therein. It is worth mentioning that, for nonlinear systems with known polynomial-of-output growth rate, the problem of output feedback disturbance attenuation was studied in [5].

In this paper, we consider output feedback disturbance attenuation problem of uncertain nonlinear systems as follows:

$$
\begin{aligned}
\dot{x}_{i}(t)= & x_{i+1}(t)+f_{i}\left(\theta^{*}(t), x(t), x\left(t-\tau_{i}(t)\right)\right) \\
& +\varphi_{i}\left(\theta^{*}(t), x(t)\right)^{\top} \omega(t), \quad i=1, \cdots, n-1, \\
\dot{x}_{n}(t)= & u(t)+v+f_{n}\left(\theta^{*}(t), x(t), x\left(t-\tau_{n}(t)\right)\right) \\
& +\varphi_{n}\left(\theta^{*}(t), x(t)\right)^{\top} \omega(t), \\
y(t)= & x_{1}(t),
\end{aligned}
$$

where $x=\left[x_{1}, \cdots, x_{n}\right]^{\top} \in R^{n}, u \in R$, and $y \in R$ are the system state, control input, and output, respectively. $v \in R$ is an unknown constant, representing the input matching uncertainty, and let $x_{n+1}=v$ for notational convenience. $\theta^{*}(t)$ : $R^{+} \longrightarrow R^{m}$ is a vector of continuous time-varying parameters belonging to an unknown bounded set. $\tau_{i}(t): R^{+} \longrightarrow$ $R^{+}, i=1, \cdots, n$, represent the bounded time-varying delays satisfying $\dot{\tau}_{i}(t) \leq \kappa<1$ for an unknown positive constant $\kappa$, and the initial condition is $x\left(t_{0}\right)=\Psi\left(t_{0}\right), t_{0} \in[-\bar{\tau}, 0]$ with $\bar{\tau}=\max _{i=1, \cdots, n}\left\{\tau_{i}(t), t \geq 0\right\}$ and $\Psi$ being a continuous function vector defined on $[-\bar{\tau}, 0] . \omega(t): R^{+} \longrightarrow R^{s}$ is disturbance satisfying $\omega(t) \in L_{2}[0,+\infty)$. For $i=1, \cdots, n$, functions $f_{i}(\cdot)$ and function vectors $\varphi_{i}(\cdot)$ are continuous in the first argument and locally Lipschitz with respect to the rest of variables.

Assumption 1. For $i=1, \cdots, n$, there is an unknown positive constant $\theta$ and a known positive integer $p \geq 1$ such that

$$
\begin{aligned}
\left|f_{i}(\cdot)\right| \leq & \theta\left(1+|y(t)|^{p}\right) \sum_{j=1}^{i}\left|x_{j}(t)\right| \\
& +\theta\left(1+\left|y\left(t-\tau_{i}(t)\right)\right|^{p}\right) \sum_{j=1}^{i}\left|x_{j}\left(t-\tau_{i}(t)\right)\right|,
\end{aligned}
$$




$$
\left\|\varphi_{i}(\cdot)\right\| \leq \theta\left(1+|y(t)|^{p}\right)
$$

During the past decade years, the problem of global output feedback control for uncertain nonlinear or nonlinear time-delay systems with unknown growth rate has been extensively studied with the aid of the dynamic gain technique, and a series of interesting results have been obtained; see [6-11] and the references therein. Specifically, for nonlinear time-delay systems with unknown polynomialof-output growth rate, [10] achieved the global output feedback stabilization based on only one dynamic gain.

However, these results do not consider the input matching uncertainty. In many practical control systems, since input matching uncertainty often causes instability or serious deterioration in the performance of systems, output feedback control of nonlinear systems with input matching uncertainty is an attractive topic in recent years; see [12-17] and the references therein. Reference [12] achieved global output feedback regulation of nonlinear systems with zero dynamics and input matching uncertainty, whose nonlinearities are bounded by unmeasured states multiplying known function of output growth rate. References $[13,14]$ investigated the problem of global adaptive output feedback stabilization of nonlinear systems with input matching uncertainty, whose uncertain nonlinearities only depend on system output. For a class of uncertain time-varying nonlinear systems with input matching uncertainty, whose nonlinearities are strictly restricted, [15] achieved global output feedback stabilization based on two dynamic gains. Lately, a compact design scheme for nonlinear systems with unknown polynomial-of-output growth rate and input matching uncertainty was proposed in [16] based on only one dynamic gain. Reference [17] studied the output tracking problem for a class of stochastic nonlinear systems with unstable modes.

As far as we know, the problem of output feedback disturbance attenuation of uncertain nonlinear systems with input matching uncertainty and unknown multiple time-varying delays, whose nonlinearities are bounded by unmeasured states multiplying unknown constant and polynomial-ofoutput growth rates, has not yet been considered until now. In this paper, we make an attempt to handle this interesting problem by skillfully combining extended state observer, dynamic gain technique, and Lyapunov-Krasovskii theorem.

Since there simultaneously exist three types of uncertainties in system (1) for the problem of disturbance attenuation, input matching uncertainty, two types of growth rates (unknown constant and polynomial-of-output growth rates), and unknown multiple time-varying delays, some essential technical difficulties to control design will be inevitably produced. (i) The observer in $[5,10]$ is inapplicable to systems of this paper due to the existence of input matching uncertainty, so a rather difficult work is how to construct a feasible observer. (ii) The analysis method in [16] is unsuitable due to the existence of unknown multiple time-varying delays; hence, another difficulty is how to give a new analysis method. This paper will focus on solving these two difficulties.

This paper is organized as follows. Section 2 gives preliminaries. In Section 3, the design and analysis of output feedback controller are presented, following a simulation example in Section 4. Section 5 concludes this paper.

\section{Mathematical Preliminaries}

In this paper, the argument of function will be omitted whenever no confusion can arise from the context. $R, R^{+}$, and $R^{n}$ denote the set of real numbers, the set of all nonnegative real numbers, and the real $n$-dimensional space, respectively. For any real vector or matrix $A, A^{\top}$ denotes its transpose; $A>0$ denotes that matrix $A$ is a positive definite matrix; $\lambda_{\text {min }}(A)$ denotes the minimal eigenvalue of the symmetric matrix $A$. For any vector $x,\|x\|_{1}$ and $\|x\|$ denote its 1-norm and 2-norm, respectively. Clearly, $\|x\| \leq\|x\|_{1} \leq \sqrt{n}\|x\|$, where $n$ is the dimension of $x$. $\operatorname{diag}\left\{a_{1}, \cdots, a_{n}\right\}$ denotes $n \times n$ diagonal matrix whose element $(k, k)$ is $a_{k}$ and others are zero. $I_{n}$ denotes the $n$-dimensional identity matrix. $L_{2}[0, T)$ and $L_{\infty}[0, T)$ denote the appropriate dimension space of square integrable functions and the appropriate dimension space of uniformly bounded functions on $[0, T)$, respectively, where $0<T \leq+\infty$.

Lemma 2 (see [12]). For any positive real number $\mu$, there exist real number $\sigma_{1}>0$, symmetric positive definite matrices $P$ and $Q$, and column vectors $a=\left[a_{1}, \cdots, a_{n+1}\right]^{\top}$ and $k=$ $\left[k_{1}, \cdots, k_{n}\right]^{\top}$ satisfying the following set of inequalities:

$$
\begin{aligned}
A^{\top} P+P A & \leq-\sigma_{1} I_{n+1}, \\
D_{n+1} P+P D_{n+1} & \geq \mu P, \\
K^{\top} Q+Q K & \leq-2 \sigma_{1} I_{n}, \\
D_{n} Q+Q D_{n} & \geq \mu Q,
\end{aligned}
$$

where $D_{i}=\operatorname{diag}\{\mu, 1+\mu, \cdots, i-1+\mu\}, i=n, n+1$, and

$$
\begin{aligned}
A & =\left[\begin{array}{cccc}
-a_{1} & 1 & \cdots & 0 \\
\vdots & \vdots & \ddots & \vdots \\
-a_{n} & 0 & \cdots & 1 \\
-a_{n+1} & 0 & \cdots & 0
\end{array}\right], \\
K & =\left[\begin{array}{cccc}
0 & 1 & \cdots & 0 \\
\vdots & \vdots & \ddots & \vdots \\
0 & 0 & \cdots & 1 \\
-k_{1} & -k_{2} & \cdots & -k_{n}
\end{array}\right] .
\end{aligned}
$$

Lemma 3 (Young's inequality). Let real numbers $p \geq 1$ and $q \geq 1$ satisfy $1 / p+1 / q=1$, then for any $x, y \in R$ and any given positive number $\gamma>0, x y \leq \gamma\|x\|^{p}+(1 / q)(p \gamma)^{-q / p}\|y\|^{q}$.

Lemma 4 (see [18]). For $x, y \in R$ and $p \geq 1$ is a constant, then $|x+y|^{p} \leq 2^{p-1}\left|x^{p}+y^{p}\right|$. 
Lemma 5 (Barbalat's lemma, see [19]). For a continuously differentiable function $x(t): R^{+} \longrightarrow R$, if $x(t), \dot{x}(t) \in L_{\infty}$ and $x(t) \in L_{p}$ for some $p \in[1,+\infty)$, then $\lim _{t \rightarrow \infty} x(t)=0$.

\section{Design and Analysis of Output Feedback Controller}

3.1. Control Objective of This Paper. The objective of this paper is to construct an output feedback controller for system (1) under Assumption 1 such that, by suitably choosing the design parameters,

(i) when $\omega(t)=0$ or $\omega(t) \in L_{2}[0,+\infty) \cap L_{\infty}[0,+\infty)$, all the states of the closed-loop system are bounded and the original system states and their corresponding observer states all converge to zero, and the estimation of the input matching uncertainty converges to its actual value.

(ii) when $\omega(t) \in L_{2}[0,+\infty)$, for any pregiven small real number $\pi>0$, the system output $y$ has the following property:

$$
\int_{0}^{t} y^{2}(s) \mathrm{d} s \leq \pi^{2} \int_{0}^{t}\|\omega(s)\|^{2} \mathrm{~d} s+\rho(\cdot),
$$

where $\rho(\cdot)$ is a nonnegative bounded function.

Remark 6. Compared with the problem of disturbance attenuation of free-delay systems in [5],

$$
\begin{aligned}
& \left|\psi_{i}(\cdot)\right| \leq \theta\left(1+|y(t)|^{p}\right) \sum_{j=1}^{i}\left|x_{j}(t)\right|, \\
& \left\|\varphi_{i}(\cdot)\right\| \leq \theta\left(1+|y(t)|^{p}\right),
\end{aligned}
$$

where $\theta$ is a known constant, and compared with the problem of stabilization control of time-delay systems in [10],

$$
\begin{aligned}
\left|\phi_{i}(\cdot)\right| \leq & \theta\left(1+|y(t)|^{p}\right) \sum_{j=1}^{i}\left|x_{j}(t)\right| \\
& +\theta\left(1+|y(t-d(t))|^{p}\right) \sum_{j=1}^{i}\left|x_{j}(t-d(t))\right|,
\end{aligned}
$$

where $d(t)$ is an unknown time-varying delay; it is worth mentioning that none of the systems in $[5,10]$ take into account the input match uncertainty. Furthermore, compared with the problem of stabilization control of free-delay systems in [16] with input matching uncertainty,

$$
\left|f_{i}(\cdot)\right| \leq \theta\left(1+|y(t)|^{p}\right) \sum_{j=1}^{i}\left|x_{j}(t)\right|,
$$

where $\theta$ is an unknown constant. This paper considers the problem of disturbance attenuation for the case in which there simultaneously exist input matching uncertainty, unknown polynomial-of-output growth rate, and unknown multiple time-varying delays; all these factors lead to some essential technical difficulties to control design of the more general systems in this paper.
3.2. The Design of Observer and Controller for System (1). Motivated by [14-16], we design the following extended state observer to rebuild the unmeasured states and estimate the input matching uncertainty and construct a coupled controller:

$$
\begin{aligned}
\dot{\hat{x}}_{i} & =\widehat{x}_{i+1}+a_{i} L^{i}\left(x_{1}-\widehat{x}_{1}\right), \quad i=1, \cdots, n-1, \\
\dot{\hat{x}}_{n} & =u+\widehat{x}_{n+1}+a_{n} L^{n}\left(x_{1}-\widehat{x}_{1}\right), \\
\dot{\hat{x}}_{n+1} & =a_{n+1} L^{n+1}\left(x_{1}-\widehat{x}_{1}\right), \\
u & =-L^{n} k_{1} \widehat{x}_{1}-L^{n-1} k_{2} \widehat{x}_{2}-\cdots-L k_{n} \widehat{x}_{n}-\widehat{x}_{n+1},
\end{aligned}
$$

with $L$ being a dynamic gain updated by

$$
\begin{aligned}
& \dot{L}(t)=\max \left\{-\rho_{1} L^{2}(t)+\rho_{2} L(t)\right. \\
& \left.\cdot\left(1+|y(t)|^{p}\right)^{2}, L^{1-2 \mu}(t)\left(x_{1}(t)-\widehat{x}_{1}(t)\right)^{2}\right\}, \\
& L(0)=1,
\end{aligned}
$$

where $\hat{x}=\left[\widehat{x}_{1}, \cdots, \widehat{x}_{n}\right]^{\top} \in R^{n}$ and $\widehat{x}_{n+1} \in R$ are the observer states. $\mu>0$ is a design parameter and will be first selected such that $0<\mu<1 / 2 p, p$ is the same as in Assumption 1. Then, according to Lemma 2 , a set $\left(\sigma_{1}, P, Q, a, k\right)$ can be determined to satisfy the inequalities in Lemma 2 , and the vectors $a$ and $k$ are selected as the gains of the extended state observer and controller, respectively. $\rho_{1}$ and $\rho_{2}$ are positive design parameters to be determined. The dynamic gain $L(t)$ has the following properties for all $t \geq 0$ :

$$
\begin{aligned}
& \dot{L}(t) \geq 0, \\
& L(t) \geq 1, \\
& L(t) \geq L\left(t-\tau_{i}(t)\right), \quad i=1, \cdots, n, \\
& \dot{L}(t) \geq-\rho_{1} L^{2}(t)+\rho_{2} L(t)\left(1+|y(t)|^{p}\right)^{2}, \\
& \dot{L}(t) \geq L^{1-2 \mu}(t)\left(x_{1}(t)-\widehat{x}_{1}(t)\right)^{2} .
\end{aligned}
$$

Remark 7. Since the existence of the input matching uncertainty $v$ in system (1), the introduction of $\widehat{x}_{n+1}$ in the observer (9) is indispensable to compensate the input matching uncertainty $v$. In Theorem 8 , we will prove that $\widehat{x}_{n+1}$ ultimately converge to the actual value of the input matching uncertainty $v$.

Introduce coordinate transformation

$$
\begin{aligned}
& \varepsilon_{i}=\frac{x_{i}-\widehat{x}_{i}}{L^{i-1+\mu}}, \quad i=1, \cdots, n+1, \\
& z_{i}=\frac{\widehat{x}_{i}}{L^{i-1+\mu}}, \quad i=1, \cdots, n .
\end{aligned}
$$

By (1), (9), and (12), we obtain

$$
\begin{aligned}
& \dot{\varepsilon}=L A \varepsilon+F-\frac{\dot{L}}{L} D_{n+1} \varepsilon, \\
& \dot{z}=L K z+L a^{*} \varepsilon_{1}-\frac{\dot{L}}{L} D_{n} z,
\end{aligned}
$$


where $\varepsilon=\left[\varepsilon_{1}, \cdots, \varepsilon_{n+1}\right]^{\top}, z=\left[z_{1}, \cdots, z_{n}\right]^{\top}, F=\left[\left(f_{1}+\right.\right.$ $\left.\left.\varphi_{1}^{\top} \omega\right) / L^{\mu}, \cdots,\left(f_{n}+\varphi_{n}^{\top} \omega\right) / L^{n-1+\mu}, 0\right]^{\top}, a^{*}=\left[a_{1}, \cdots, a_{n}\right]^{\top}$. Let $V_{\varepsilon}=m \varepsilon^{\top} P \varepsilon$ and $V_{z}=z^{\top} Q z$ with $m=1+\left\|Q a^{*}\right\|^{2} / \sigma_{1}^{2}$. By (11), (13), Lemma 2 , and the fact that $\lambda_{\min }(P)\|\varepsilon\|^{2} \leq \varepsilon^{\top} P \varepsilon$ and $\lambda_{\text {min }}(Q)\|z\|^{2} \leq z^{\top} Q z$, we have

$$
\begin{aligned}
\dot{V}_{\varepsilon} \leq & -\sigma_{1} m L\|\varepsilon\|^{2}+2 m \varepsilon^{\top} P F+m \rho_{1} \mu \lambda_{\min }(P) L\|\varepsilon\|^{2} \\
& -m \rho_{2} \mu \lambda_{\min }(P)\left(1+|y|^{p}\right)^{2}\|\varepsilon\|^{2} \\
\dot{V}_{z} \leq & -2 \sigma_{1} L\|z\|^{2}+2 L z^{\top} Q a^{*} \varepsilon_{1}+\rho_{1} \mu \lambda_{\min }(Q) L\|z\|^{2} \\
& -\rho_{2} \mu \lambda_{\min }(Q)\left(1+|y|^{p}\right)^{2}\|z\|^{2} .
\end{aligned}
$$

By Lemma 3, it is easy to get

$$
2 L z^{\top} Q a^{*} \varepsilon_{1} \leq \sigma_{1} L\|z\|^{2}+\frac{\left\|Q a^{*}\right\|^{2}}{\sigma_{1}} L \varepsilon_{1}^{2} .
$$

By (2), (12), and the fact that $L(t) \geq 1$ is a monotone nondecreasing function for $i=1, \cdots, n$,

$$
\begin{aligned}
& \left|\frac{f_{i}+\varphi_{i}^{\top} \omega}{L^{i-1+\mu}}\right| \leq \frac{\theta\left(1+|y|^{p}\right)}{L^{i-1+\mu}}\left(\sum_{j=1}^{i}\left|x_{j}\right|+\|\omega\|\right) \\
& \quad+\frac{\theta\left(1+\left|y\left(t-\tau_{i}(t)\right)\right|^{p}\right)}{L^{i-1+\mu}} \sum_{j=1}^{i}\left|x_{j}\left(t-\tau_{i}(t)\right)\right| \\
& \quad \leq \theta\left(1+|y|^{p}\right) \sqrt{n}(\|\varepsilon\|+\|z\|)+\theta\left(1+|y|^{p}\right)\|\omega\| \\
& +\theta\left(1+\left|y\left(t-\tau_{i}(t)\right)\right|^{p}\right) \\
& \cdot \sqrt{n}\left(\left\|\varepsilon\left(t-\tau_{i}(t)\right)\right\|+\left\|z\left(t-\tau_{i}(t)\right)\right\|\right) .
\end{aligned}
$$

Using (17) and Lemma 3, it follows that

$$
\begin{aligned}
& 2 m \varepsilon^{\top} P F \leq 2 m\|P\|\|\varepsilon\| \sum_{i=1}^{n}\left|\frac{f_{i}+\varphi_{i}^{\top} \omega}{L^{i-1+\mu}}\right| \leq\left(1+|y|^{p}\right)^{2} \\
& \cdot\left(\|\varepsilon\|^{2}+\|z\|^{2}\right)+d_{0}\|\varepsilon\|^{2}+g_{0}\|\omega\|^{2}+\sum_{i=1}^{n} \frac{1-\kappa}{n} \\
& \cdot\left(1+\left|y\left(t-\tau_{i}(t)\right)\right|^{p}\right)^{2} \\
& \cdot\left(\left\|\varepsilon\left(t-\tau_{i}(t)\right)\right\|^{2}+\left\|z\left(t-\tau_{i}(t)\right)\right\|^{2}\right),
\end{aligned}
$$

where $d_{0}=(2+2 /(1-\kappa)) n^{3} m^{2} \theta^{2}\|P\|^{2}$ and $g_{0}=2 n^{2} m^{2} \theta^{2}\|P\|^{2}$ are unknown positive constants related to $\theta$ and $\kappa$.

Choose the Lyapunov-Krasovskii functional

$$
\begin{gathered}
V=V_{\varepsilon}+V_{z}+\sum_{i=1}^{n} \int_{t-\tau_{i}(t)}^{t} \frac{1}{n}\left(1+|y(s)|^{p}\right)^{2} \\
\cdot\left(\|\varepsilon(s)\|^{2}+\|z(s)\|^{2}\right) \mathrm{d} s,
\end{gathered}
$$

and select the design parameters $\rho_{1}$ and $\rho_{2}$ to satisfy

$$
\begin{aligned}
& \rho_{1} \leq \min \left\{\frac{\sigma_{1}}{2 m \mu \lambda_{\text {min }}(P)}, \frac{\sigma_{1}}{2 \mu \lambda_{\text {min }}(Q)}\right\}, \\
& \rho_{2} \geq \max \left\{\frac{2}{m \mu \lambda_{\text {min }}(P)}, \frac{2}{\mu \lambda_{\text {min }}(Q)}\right\} .
\end{aligned}
$$

Then, using (14)-(16), (18)-(20), and $\dot{t}_{i}(t) \leq \kappa<1, i=$ $1, \cdots, n$, we arrive at

$$
\dot{V} \leq-\frac{\sigma_{1}}{2} L\left(\|\varepsilon\|^{2}+\|z\|^{2}\right)+d_{0}\|\varepsilon\|^{2}+g_{0}\|\omega\|^{2} .
$$

3.3. Stability and Convergence Analysis. We state the main result in this paper.

Theorem 8. Consider system (1) satisfying Assumption 1, and under the output feedback controller (9) and (10), the closed-loop system consisting of (1), (9), and (10) achieves global disturbance attenuation in the $L_{2}$-gain sense. Moreover, if $\omega(t)=0$ or $\omega(t) \in L_{2}[0,+\infty) \cap L_{\infty}[0,+\infty)$, then $\lim _{t \rightarrow+\infty}\left(x(t), \widehat{x}(t), \widehat{x}_{n+1}, u(t)\right)=(0,0, v,-v)$.

Proof. It is observed that the right-hand side of the closedloop system consisting of (1), (9), and (10) is continuous and locally Lipschitz in $\left(x, \widehat{x}, \widehat{x}_{n+1}, u\right)$; hence, the closed-loop system has a unique solution on the maximal interval $\left[0, t_{f}\right)$ with $0<t_{f} \leq+\infty$. Next, we divide the proof into two steps.

Step I (the boundedness of $L(t), \varepsilon(t)$, and $z(t)$ on $\left[0, t_{f}\right)$ )

(i) Boundedness of $L(t)$ on $\left[0, t_{f}\right)$. We prove the boundedness of $L(t)$ on $\left[0, t_{f}\right)$ by a contradiction argument. Suppose that $L(t)$ is unbounded on $\left[0, t_{f}\right)$; note the monotone nondecreasing property of $L(t)$; there holds $\lim _{t \rightarrow t_{f}} L(t)=+\infty$. Hence, there is a finite time $t_{1} \in\left[0, t_{f}\right)$ such that $L(t) \geq$ $\max \left\{1,4 d_{0} / \sigma_{1}\right\}, \forall t \in\left[t_{1}, t_{f}\right)$. Then, from (21), it follows that

$$
\begin{aligned}
\dot{V}(t) \leq & -\frac{\sigma_{1}}{4} L(t)\|\mathcal{\varepsilon}(t)\|^{2}-\frac{\sigma_{1}}{2} L(t)\|z(t)\|^{2} \\
& +g_{0}\|\omega(t)\|^{2}, \quad \forall t \in\left[t_{1}, t_{f}\right),
\end{aligned}
$$

which, together with $\omega(t) \in L_{2}[0,+\infty)$, implies that $\varepsilon(t)$ and $z(t)$ are bounded on $\left[t_{1}, t_{f}\right)$ and

$$
\begin{aligned}
& \frac{\sigma_{1}}{4} \int_{t_{1}}^{t_{f}} L(s)\|\varepsilon(s)\|^{2} \mathrm{~d} s \leq V\left(t_{1}\right)+g_{0} \int_{t_{1}}^{t_{f}}\|\omega(s)\|^{2} \mathrm{~d} s \\
& \quad<+\infty .
\end{aligned}
$$

By (12), Lemma 4 , and the fact that $L(t) \geq 1$, it is obvious that

$$
\begin{aligned}
- & \rho_{1} L^{2}(t)+\rho_{2} L(t)\left(1+|y(t)|^{p}\right)^{2} \\
\leq & -\rho_{1} L^{2}(t) \\
& +2 \rho_{2}\left(1+\left|\varepsilon_{1}(t)+z_{1}(t)\right|^{2 p}\right) L^{1+2 \mu p}(t) .
\end{aligned}
$$

Then, by $0<\mu<1 / 2 p$ and the boundedness of $\varepsilon(t)$ and $z(t)$ on $\left[t_{1}, t_{f}\right)$, there is a finite time $t_{2} \in\left[t_{1}, t_{f}\right)$, such that 
$-\rho_{1} L^{2}(t)+\rho_{2} L(t)\left(1+|y(t)|^{p}\right)^{2} \leq 0$ on $\left[t_{2}, t_{f}\right)$, which, together with (10) and (12), implies that

$$
\begin{aligned}
+\infty & =L\left(t_{f}\right)-L\left(t_{2}\right)=\int_{t_{2}}^{t_{f}} \dot{L}(s) \mathrm{d} s \\
& =\int_{t_{2}}^{t_{f}} L(s) \varepsilon_{1}^{2}(s) \mathrm{d} s \leq \int_{t_{1}}^{t_{f}} L(s)\|\varepsilon(s)\|^{2} \mathrm{~d} s .
\end{aligned}
$$

This contradicts with (23). Thus, $L(t)$ is bounded on $\left[0, t_{f}\right)$ and suppose $\lim _{t \rightarrow t_{f}} L(t)=\bar{L}$ with $\bar{L} \geq 1$ being a constant.

(ii) Boundedness of $z(t)$ on $\left[0, t_{f}\right)$. By (11)-(12), (15)-(16), and (20), we obtain

$$
\begin{aligned}
\dot{V}_{z} \leq & -\frac{\sigma_{1}}{2}\|z\|^{2}+\frac{\left\|Q a^{*}\right\|^{2}}{\sigma_{1}} \dot{L} \\
& -\rho_{2} \mu \lambda_{\min }(\mathrm{Q})\left(1+|y|^{p}\right)^{2}\|z\|^{2} .
\end{aligned}
$$

Integrating both sides of (26) with $L(t) \geq 1$ being a monotone nondecreasing function and $L(0)=1, \lim _{t \rightarrow t_{f}} L(t)=\bar{L}$ leads to, on $\left[0, t_{f}\right)$,

$$
\begin{aligned}
\lambda_{\min }(Q)\|z(t)\|^{2} \leq & V_{z}(t) \\
\leq & V_{z}(0)-\frac{\sigma_{1}}{2} \int_{0}^{t}\|z(s)\|^{2} \mathrm{~d} s \\
& +\frac{\left\|Q a^{*}\right\|^{2}}{\sigma_{1}}(\bar{L}-1),
\end{aligned}
$$

from which it follows that, $\forall t \in\left[0, t_{f}\right)$,

$$
\begin{aligned}
\|z(t)\|^{2} & \leq \frac{1}{\lambda_{\min }(Q)}\left(V_{z}(0)+\frac{\left\|Q a^{*}\right\|^{2}}{\sigma_{1}} \bar{L}\right), \\
\int_{0}^{t}\|z(s)\|^{2} \mathrm{~d} s & \leq \frac{2}{\sigma_{1}}\left(V_{z}(0)+\frac{\left\|Q a^{*}\right\|^{2}}{\sigma_{1}} \bar{L}\right),
\end{aligned}
$$

which implies that $z(t)$ and $\int_{0}^{t}\|z(s)\|^{2} \mathrm{~d} s$ are bounded on $\left[0, t_{f}\right)$.

(iii) Boundedness of $\varepsilon(t)$ on $\left[0, t_{f}\right)$. To prove the boundedness of $\varepsilon(t)$ on $\left[0, t_{f}\right)$, we introduce a new change of coordinate

$$
\epsilon_{i}=\frac{\varepsilon_{i}}{\left(L^{*}\right)^{i-1+\mu}}, \quad i=1, \cdots, n+1,
$$

where the constant is $L^{*} \geq 1 / m+2 d_{1} / \sigma_{1}+1>1$ with $d_{1}$ being a positive constant to be defined. By (13) and (29), we arrive at

$$
\dot{\epsilon}=L L^{*} A \epsilon+L L^{*} a \epsilon_{1}-L \Gamma a \epsilon_{1}+F^{*}-\frac{\dot{L}}{L} D_{n+1} \epsilon,
$$

where $\epsilon=\left[\epsilon_{1}, \cdots, \epsilon_{n+1}\right]^{\top}, \Gamma=\operatorname{diag}\left\{1,1 / L^{*}, \cdots, 1 /\left(L^{*}\right)^{n}\right\}$, and $F^{*}=\left[\left(f_{1}+\varphi_{1}^{\top} \omega\right) /\left(L L^{*}\right)^{\mu}, \cdots,\left(f_{n}+\varphi_{n}^{\top} \omega\right) /\left(L L^{*}\right)^{n-1+\mu}, 0\right]^{\top}$.
Choose the Lyapunov function $V_{\epsilon}=\epsilon^{\top} P \epsilon$. By (11), (30), Lemma 2, and the fact that $\lambda_{\min }(P)\|\epsilon\|^{2} \leq \epsilon^{\top} P \epsilon$,

$$
\begin{aligned}
\dot{V}_{\epsilon} \leq & -\sigma_{1} L L^{*}\|\epsilon\|^{2}+2 L L^{*} \epsilon^{\top} P a \epsilon_{1}-2 L \epsilon^{\top} P \Gamma a \epsilon_{1} \\
& +2 \epsilon^{\top} P F^{*}+L \rho_{1} \mu \lambda_{\min }(P)\|\epsilon\|^{2} \\
& -\rho_{2} \mu \lambda_{\min }(P)\left(1+|y|^{p}\right)^{2}\|\epsilon\|^{2} .
\end{aligned}
$$

Using (11), (12), (29), and Lemma 3, we obtain

$$
\begin{aligned}
& 2 L L^{*} \epsilon^{\top} P a \epsilon_{1} \leq \frac{\sigma_{1} L L^{*}}{4}\|\epsilon\|^{2}+\frac{4\left(L^{*}\right)^{1-2 \mu}\|P a\|^{2}}{\sigma_{1}} \dot{L}, \\
& -2 L \epsilon^{\top} P \Gamma a \epsilon_{1} \leq \frac{\sigma_{1} L L^{*}}{4}\|\epsilon\|^{2}+\frac{4\|P \Gamma a\|^{2}}{\sigma_{1}\left(L^{*}\right)^{1+2 \mu}} \dot{L} .
\end{aligned}
$$

Similar to the proof of (18) with $L^{*} \geq 1$, one gets

$$
\begin{gathered}
2 \epsilon^{\top} P F^{*} \leq\left(1+|y|^{p}\right)^{2}\left(\frac{1}{m}\|\epsilon\|^{2}+\|z\|^{2}\right)+d_{1}\|\epsilon\|^{2} \\
+g_{1}\|\omega\|^{2}+\sum_{i=1}^{n} \frac{1-\kappa}{n}\left(1+\left|y\left(t-\tau_{i}(t)\right)\right|^{p}\right)^{2} \\
\cdot\left(\frac{1}{m}\left\|\epsilon\left(t-\tau_{i}(t)\right)\right\|^{2}+\left\|z\left(t-\tau_{i}(t)\right)\right\|^{2}\right),
\end{gathered}
$$

where $d_{1}=n^{3} \theta^{2}\|P\|^{2}(2 m+1+(m+1) /(1-\kappa)), g_{1}=$ $2 m n^{2} \theta^{2}\|P\|^{2}$ are unknown positive constants related to $\theta$ and $\kappa$.

Choose the Lyapunov-Krasovskii functional

$$
\begin{aligned}
& V^{*}=V_{\epsilon}+V_{z}+\sum_{i=1}^{n} \int_{t-\tau_{i}(t)}^{t} \frac{1}{n}\left(1+|y(s)|^{p}\right)^{2} \\
& \cdot\left(\frac{1}{m}\|\epsilon(s)\|^{2}+\|z(s)\|^{2}\right) \mathrm{d} s .
\end{aligned}
$$

By (20), (26), (31)-(34), the definition of $L^{*}$, and the fact that $L(t) \geq 1$, we derive

$$
\begin{aligned}
\dot{V}^{*} \leq & -\frac{\sigma_{1}}{2} L L^{*}\|\epsilon\|^{2}+L \rho_{1} \mu \lambda_{\text {min }}(P)\|\epsilon\|^{2} \\
& -\rho_{2} \mu \lambda_{\text {min }}(P)\left(1+|y|^{p}\right)^{2}\|\epsilon\|^{2} \\
& +\frac{2}{m}\left(1+|y|^{p}\right)^{2}\|\varepsilon\|^{2}+\eta \dot{L}+2\left(1+|y|^{p}\right)^{2}\|z\|^{2} \\
& +d_{1}\|\epsilon\|^{2}+g_{1}\|\omega\|^{2} \\
& -\rho_{2} \mu \lambda_{\min }(Q)\left(1+|y|^{p}\right)^{2}\|z\|^{2}+\frac{\sigma_{1}}{2 m} L\|\epsilon\|^{2} \\
& -\frac{\sigma_{1}}{2 m} L\|\epsilon\|^{2} \\
\leq & -\frac{\sigma_{1}}{2} L\left(L^{*}-\frac{1}{m}-\frac{2 d_{1}}{\sigma_{1}}\right)\|\epsilon\|^{2}+\eta \dot{L}+g_{1}\|\omega\|^{2} \\
\leq & -\frac{\sigma_{1}}{2}\|\epsilon\|^{2}+\eta \dot{L}+g_{1}\|\omega\|^{2},
\end{aligned}
$$


where $\eta=\left\|Q a^{*}\right\|^{2} / \sigma_{1}+4\left(L^{*}\right)^{1-2 \mu}\|P a\|^{2} / \sigma_{1}+4\|P \Gamma a\|^{2} /$ $\sigma_{1}\left(L^{*}\right)^{1+2 \mu}$ is a positive constant. Similar to the proof of (27), integrating both sides of (35) yields, on $\left[0, t_{f}\right)$,

$$
\begin{aligned}
\lambda_{\min }(P)\|\epsilon(t)\|^{2} \leq & V^{*}(t) \\
\leq & V^{*}(0)-\frac{\sigma_{1}}{2} \int_{0}^{t}\|\epsilon(s)\|^{2} \mathrm{~d} s \\
& +\eta(\bar{L}-1)+g_{1} \int_{0}^{t}\|\omega(s)\|^{2} \mathrm{~d} s,
\end{aligned}
$$

from which it follows that, $\forall t \in\left[0, t_{f}\right)$,

$$
\begin{aligned}
& \|\epsilon(t)\|^{2} \\
& \leq \frac{1}{\lambda_{\min }(P)}\left(V^{*}(0)+\eta \bar{L}+g_{1} \int_{0}^{t}\|\omega(s)\|^{2} \mathrm{~d} s\right), \\
& \int_{0}^{t}\|\epsilon(s)\|^{2} \mathrm{~d} s \\
& \quad \leq \frac{2}{\sigma_{1}}\left(V^{*}(0)+\eta \bar{L}+g_{1} \int_{0}^{t}\|\omega(s)\|^{2} \mathrm{~d} s\right),
\end{aligned}
$$

which together with $\omega(t) \in L_{2}[0,+\infty)$ implies that $\epsilon(t)$ and $\int_{0}^{t}\|\epsilon(s)\|^{2} \mathrm{~d} s$ are bounded on $\left[0, t_{f}\right)$. From (29) with the fact that $L^{*}$ is a constant, we obtain $\varepsilon(t)$ and $\int_{0}^{t}\|\varepsilon(s)\|^{2} \mathrm{~d} s$ are bounded on $\left[0, t_{f}\right)$.

Step II (we prove that the closed-loop system consisting of (1), (9), and (10) achieves global disturbance attenuation in the $L_{2}$-gain sense). From (12), it is easy to get

$$
\begin{aligned}
x_{i} & =L^{i-1+\mu}\left(\varepsilon_{i}+z_{i}\right), \quad i=1, \cdots, n, \\
\widehat{x}_{i} & =L^{i-1+\mu} z_{i}, \quad i=1, \cdots, n, \\
\widehat{x}_{n+1} & =v-L^{n+\mu} \varepsilon_{n+1} .
\end{aligned}
$$

When $\omega(t) \in L_{2}[0,+\infty)$, by the boundedness of $L(t), z(t)$, $\varepsilon(t)$ on $\left[0, t_{f}\right)$ and the fact that $\nu$ is a constant, it can be derived from (38) that $x(t), \widehat{x}(t)$, and $\widehat{x}_{n+1}(t)$ are bounded on $\left[0, t_{f}\right)$. By (9), it follows that $u(t)$ is also bounded on $\left[0, t_{f}\right)$.

Then, we prove that $t_{f}=+\infty$. The conclusion follows again by a contradiction argument. Suppose $t_{f}<+\infty$, then $t_{f}$ would be the finite-escape time of the closed-loop system, which means that at least one component of $x(t), \widehat{x}(t), \widehat{x}_{n+1}(t)$, and $L(t)$ would tend to infinity at $t=t_{f}$. However, $x(t), \widehat{x}(t)$, $\widehat{x}_{n+1}(t)$, and $L(t)$ are bounded on the maximal interval $\left[0, t_{f}\right)$ and hence also bounded at $t=t_{f}$ due to the continuity of the solution; this is a contradiction.

$t_{f}=+\infty$ means that $L(t), z(t), \varepsilon(t), \int_{0}^{t}\|z(s)\|^{2} \mathrm{~d} s$, $\int_{0}^{t}\|\varepsilon(s)\|^{2} \mathrm{~d} s$ are bounded on $[0,+\infty)$, which indicates that $x(t), \widehat{x}(t), \widehat{x}_{n+1}(t)$, and $u(t)$ are bounded on $[0,+\infty)$, and $\int_{0}^{+\infty}\|z(s)\|^{2} \mathrm{~d} s<+\infty, \int_{0}^{+\infty}\|\varepsilon(s)\|^{2} \mathrm{~d} s<+\infty$. Meanwhile, from (38) with $y=x_{1}$, we obtain $\int_{0}^{+\infty} y^{2}(s) \mathrm{d} s<+\infty$.

Using (21), for any pregiven small real number $\pi>0$,

$$
\dot{V}+\frac{g_{0}}{\pi^{2}} y^{2} \leq d_{0}\|\varepsilon\|^{2}+g_{0}\|\omega\|^{2}+\frac{g_{0}}{\pi^{2}} y^{2} .
$$

Integrating both sides of (39) leads to, $\forall t \geq 0$,

$$
\begin{aligned}
\int_{0}^{t} y^{2}(s) \mathrm{d} s \leq & \pi^{2} \int_{0}^{t}\|\omega(s)\|^{2} \mathrm{~d} s+\frac{\pi^{2}}{g_{0}} V(0) \\
& +\frac{\pi^{2} d_{0}}{g_{0}} \int_{0}^{t}\|\varepsilon(s)\|^{2} \mathrm{~d} s+\int_{0}^{t} y^{2}(s) \mathrm{d} s
\end{aligned}
$$

choose $\rho(\cdot)=\left(\pi^{2} / g_{0}\right) V(0)+\left(\pi^{2} d_{0} / g_{0}\right) \int_{0}^{t}\|\varepsilon(s)\|^{2} \mathrm{~d} s+$ $\int_{0}^{t} y^{2}(s) \mathrm{d} s$. Obviously, $\rho(\cdot)$ is a nonnegative bounded function. Then the global disturbance attenuation of the closedloop system is achieved in the $L_{2}$-gain sense.

Moreover, if $\omega(t)=0$ or $\omega(t) \in L_{2}[0,+\infty) \cap L_{\infty}[0,+\infty)$, by the boundedness of all signals on $[0,+\infty)$, from (13), it is obvious that $\dot{\varepsilon}(t)$ and $\dot{z}(t)$ are also bounded on $[0,+\infty)$. Then, by Lemma 5 , we conclude that $\lim _{t \rightarrow+\infty}(\varepsilon(t), z(t))=(0,0)$. Therefore, from (9) and (38) with the boundedness of $L(t)$, we deduce $\lim _{t \rightarrow+\infty}\left(x(t), \widehat{x}(t), \widehat{x}_{n+1}(t), u(t)\right)=(0,0, \nu,-\nu)$.

\section{Simulation Example}

Consider a simple system

$$
\begin{aligned}
\dot{x}_{1}= & x_{2}+c_{1} x_{1}^{3}\left(t-\tau_{1}(t)\right)+c_{2} \ln \left(1+x_{1}^{2}\right) \omega, \\
\dot{x}_{2}= & u+v+c_{3} x_{1}^{2} x_{2} \\
& +c_{4} \ln \left(1+x_{1}^{2}\left(t-\tau_{2}(t)\right)\right) x_{2}\left(t-\tau_{2}(t)\right) \\
& +c_{5} \omega \\
y= & x_{1},
\end{aligned}
$$

where $v$ is an unknown constant representing the input matching uncertainty, and let $x_{3}=v \cdot c_{i}, i=1, \cdots, 5$, are unknown constants, and $\tau_{1}(t)=1+l_{1} \sin (t)$ and $\tau_{2}(t)=$ $(1 / 2) l_{2} \cos ^{2}(t)$ represent unknown time-varying delays with $\left|l_{1}\right|<1,0<l_{2}<1$ being unknown constants. The system disturbance is $\omega(t)=1 /(1+t)$; obviously, $\omega(t) \in L_{2}[0,+\infty)$. Since $\ln \left(1+x_{1}^{2}\right) \leq 1+x_{1}^{2}$, Assumption 1 holds with $\theta=$ $\max \left\{\left|c_{i}\right|, i=1, \cdots, 5\right\}, p=2$.

From Lemma 2, we choose $\mu=0.2<1 / 4, a=[3,3,1]^{\top}$, $k=[3,3]^{\top}, \sigma_{1}=18$. Then, by $(20)$, select $\rho_{1}=0.9$ and $\rho_{2}=12.8$. According to Section 3 , we get the output feedback controller

$$
\begin{aligned}
\dot{\hat{x}}_{1} & =\widehat{x}_{2}+3 L\left(x_{1}-\widehat{x}_{1}\right), \\
\dot{\hat{x}}_{2} & =\widehat{x}_{3}+u+3 L^{2}\left(x_{1}-\widehat{x}_{1}\right), \\
\dot{\hat{x}}_{3} & =L^{3}\left(x_{1}-\widehat{x}_{1}\right), \\
u & =-3 L^{2} \widehat{x}_{1}-3 L \widehat{x}_{2}-\widehat{x}_{3}, \\
\dot{L} & =\max \left\{-0.9 L^{2}\right. \\
& \left.+12.8 L\left(1+|y|^{2}\right)^{2}, L^{0.6}\left(x_{1}-\widehat{x}_{1}\right)^{2}\right\}, \quad L(0)=1 .
\end{aligned}
$$

In the simulation, we choose $c_{i}=1, i=1, \cdots, 5, v=-8$, $l_{1}=l_{2}=0.5$ and the initial values $\left[x_{1}\left(t_{0}\right), x_{2}\left(t_{0}\right)\right]=[0.5,0.8]$ 

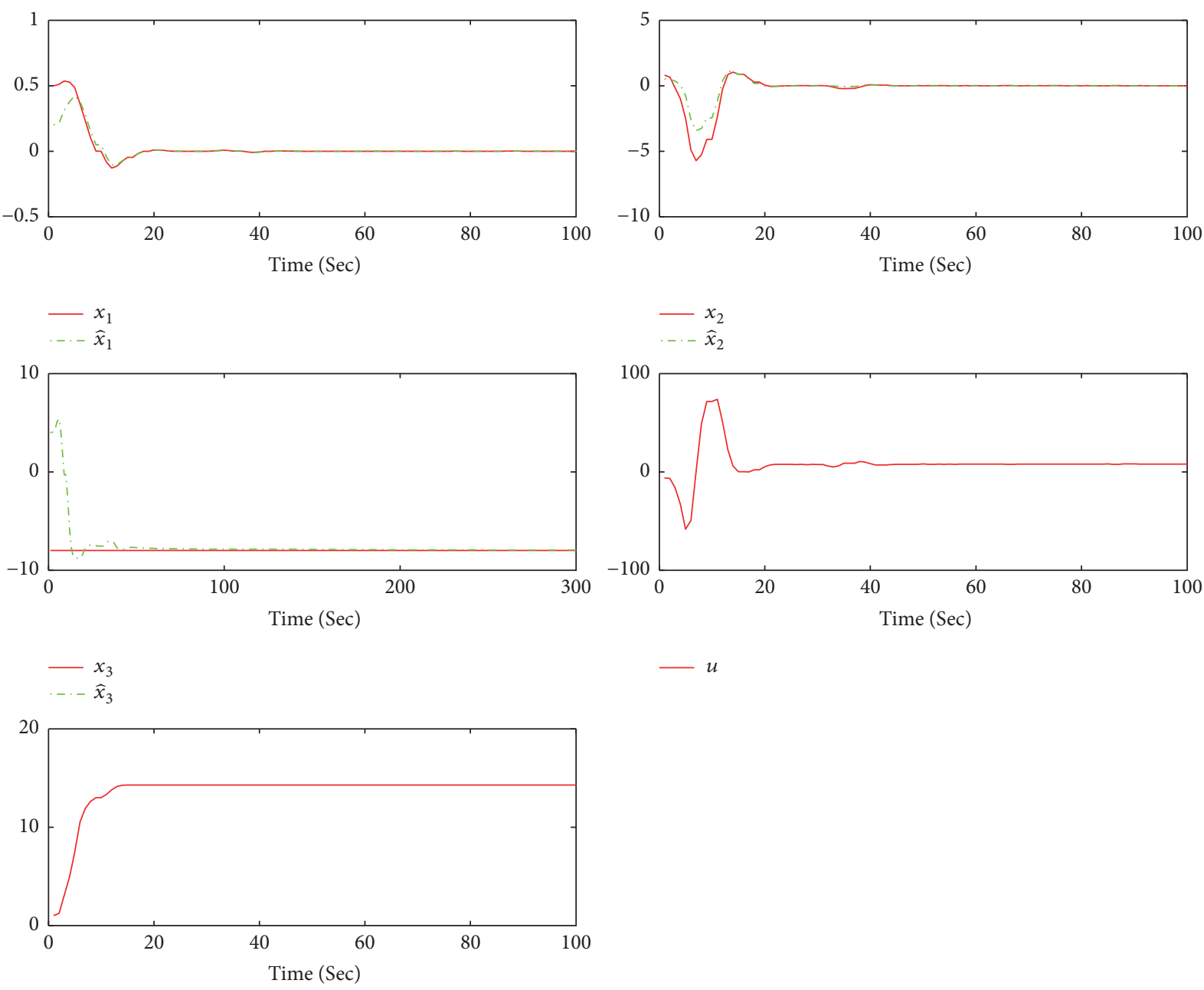

$-L$

FIGURE 1: The responses of the closed-loop system (41)-(42).

for $t_{0} \in[-1.5,0],\left[\widehat{x}_{1}(0), \widehat{x}_{2}(0), \widehat{x}_{3}(0)\right]=[0.2,0.5,4]$. Figure 1 demonstrates the effectiveness of the control scheme.

\section{Conclusions}

By skillfully combining extended state observer, dynamic gain technique, and Lyapunov-Krasovskii theorem, the problem of output feedback disturbance attenuation for nonlinear systems with input matching uncertainty and unknown multiple time-varying delays is solved in this paper based on only one dynamic gain.

Some interesting problems still remained; e.g., (i) for system (1) with the unknown output function [20-24], can we design an output feedback controller? (ii) Another work is to consider more general input matching uncertainty such as an uncertain harmonic signal.

\section{Data Availability}

The data supporting the conclusions of this manuscript are some open access articles that have been properly cited, and the readers can easily obtain these articles to verify the conclusions, replicate the analysis, and conduct secondary analyses. Therefore, we do not create a publicly available data repository.

\section{Conflicts of Interest}

The authors declare that they have no conflicts of interest.

\section{Acknowledgments}

This work was supported by the Taishan Scholar Project of Shandong Province of China (no. ts201712040), National Natural Science Foundation of China (no. 61673242), and Shandong Provincial Natural Science Foundation of China (no. ZR2016FM10).

\section{References}

[1] W. Lin, C.-J. Qian, and X.-Q. Huang, "Disturbance attenuation of a class of non-linear systems via output feedback," International Journal of Robust and Nonlinear Control, vol. 13, no. 15, pp. 1359-1369, 2003. 
[2] H. Ito and Z.-P. Jiang, "Robust disturbance attenuation of nonlinear systems using output feedback and state-dependent scaling," Automatica, vol. 40, no. 9, pp. 1621-1628, 2004.

[3] Y.-J. Pan, H.-J. Marquez, and T. Chen, "A new output feedback stabilization controller for the disturbance attenuation of MIMO non-linear systems," International Journal of Control, vol. 80, no. 9, pp. 1481-1490, 2007.

[4] F. Shang, Y. G. Liu, G. Q. Zhang, and C. H. Zhang, "Adaptive disturbance attenuation by output feedback for a class of nonlinear systems," Acta Automatica Sinica, vol. 37, no. 12, pp. 1530-1536, 2011.

[5] F. Shang and Y.-G. Liu, "Adaptive disturbance attenuation via output feedback for nonlinear systems with polynomial-ofoutput growth rate," International Journal of Control, vol. 87, no. 3, pp. 600-611, 2014.

[6] H. Lei and W. Lin, "Universal adaptive control of nonlinear systems with unknown growth rate by output feedback," Automatica, vol. 42, no. 10, pp. 1783-1789, 2006.

[7] H. Lei and W. Lin, "Adaptive regulation of uncertain nonlinear systems by output feedback: a universal control approach," Systems and Control Letters, vol. 56, no. 7-8, pp. 529-537, 2007.

[8] J.-Y. Zhai and W.-T. Zha, "Global adaptive output feedback control for a class of nonlinear time-delay systems," ISA Transactions, vol. 53, no. 1, pp. 2-9, 2014.

[9] A. Benabdallah, H. Ayadi, and M. Mabrouk, "Global adaptive output stabilization of uncertain nonlinear systems with polynomial output depending growth rate," International Journal of Adaptive Control and Signal Processing, vol. 28, no. 7-8, pp. 604619, 2014.

[10] X. Zhang and Y. Lin, "Global adaptive output feedback control for a class of uncertain nonlinear time-delay systems," in Proceedings of the 33rd Chinese Control Conference, CCC 2014, pp. 2255-2260, China, July 2014.

[11] X.-L. Jia, X.-K. Chen, S.-Y. Xu, B.-Y. Zhang, and Z.-Q. Zhang, "Adaptive output feedback control of nonlinear time-delay systems with application to chemical reactor systems," IEEE Transactions on Industrial Electronics, vol. 64, no. 6, pp. 47924799, 2017.

[12] L. Praly and Z.-P. Jiang, "Linear output feedback with dynamic high gain for nonlinear systems," Systems and Control Letters, vol. 53, no. 2, pp. 107-116, 2004.

[13] Z.-T. Ding, "Asymptotic rejection of unmatched general periodic disturbances with nonlinear Lipschitz internal models," International Journal of Control, vol. 86, no. 2, pp. 210-221, 2013.

[14] Y.-X. Huang and Y.-G. Liu, "Adaptive output-feedback control of nonlinear systems with multiple uncertainties," Asian Journal of Control, vol. 20, no. 3, pp. 1151-1160, 2018.

[15] Y.-C. Man and Y.-G. Liu, "Global output-feedback stabilization for a class of uncertain time-varying nonlinear systems," Systems and Control Letters, vol. 90, pp. 20-30, 2016.

[16] Y.-X. Huang and Y.-G. Liu, "A compact design scheme of adaptive output-feedback control for uncertain nonlinear systems," International Journal of Control, pp. 1-9, 2017.

[17] W.-Q. Li, L. Liu, and G. Feng, "Output tracking of stochastic nonlinear systems with unstable linearization," International Journal of Robust and Nonlinear Control, vol. 28, no. 2, pp. 466477, 2018.

[18] C.-J. Qian and W. Lin, "A continuous feedback approach to global strong stabilization of nonlinear systems," Institute of Electrical and Electronics Engineers Transactions on Automatic Control, vol. 46, no. 7, pp. 1061-1079, 2001.
[19] H.-K. Khalil, Nonlinear Systems, Prentice-Hall, New Jersey, NJ, USA, 2002

[20] Z.-J. Li, X.-J. Xie, and K.-M. Zhang, "Output feedback stabilisation for nonlinear systems with unknown output function and control coefficients and its application," International Journal of Control, vol. 90, no. 5, pp. 1027-1036, 2017.

[21] M.-M. Jiang, K.-M. Zhang, and X.-J. Xie, “Output feedback stabilisation of stochastic nonlinear time-delay systems with unknown output function," International Journal of Systems Science, vol. 48, no. 11, pp. 2262-2271, 2017.

[22] M.-M. Jiang, P. Wang, and X.-J. Xie, "Global output feedback stabilisation of nonlinear time-delay systems with unknown output function," International Journal of Systems Science, vol. 48, no. 12, pp. 2554-2564, 2017.

[23] X.-J. Xie, Z.-J. Li, and K.-M. Zhang, "Semi-global output feedback control for nonlinear systems with uncertain timedelay and output function," International Journal of Robust and Nonlinear Control, vol. 27, no. 15, pp. 2549-2566, 2017.

[24] X.-J. Xie and M.-M. Jiang, "Output feedback stabilization of stochastic feedforward nonlinear time-delay systems with unknown output function," International Journal of Robust and Nonlinear Control, vol. 28, no. 1, pp. 266-280, 2018. 


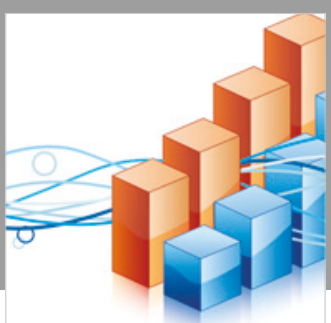

Advances in

Operations Research

\section{-n-m}
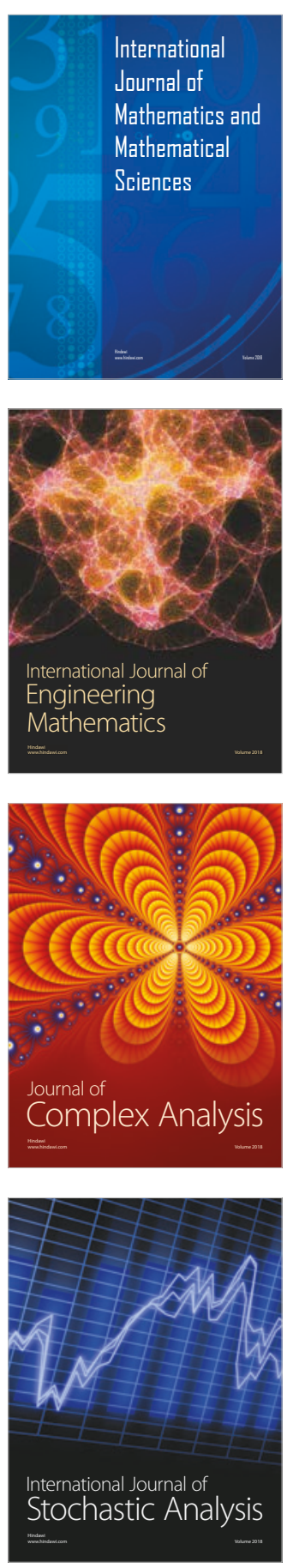
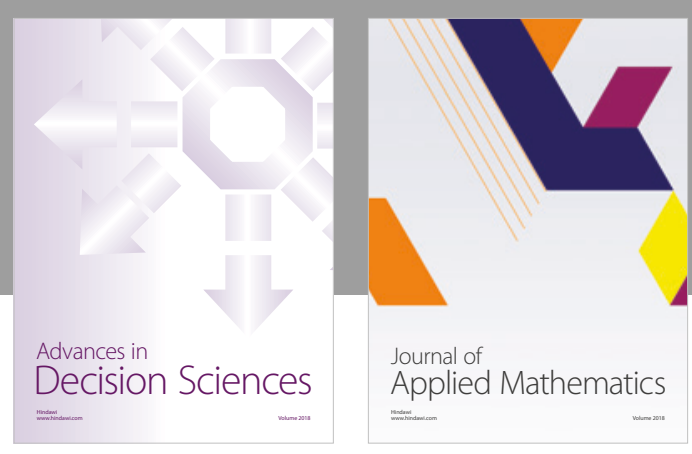

Journal of

Applied Mathematics
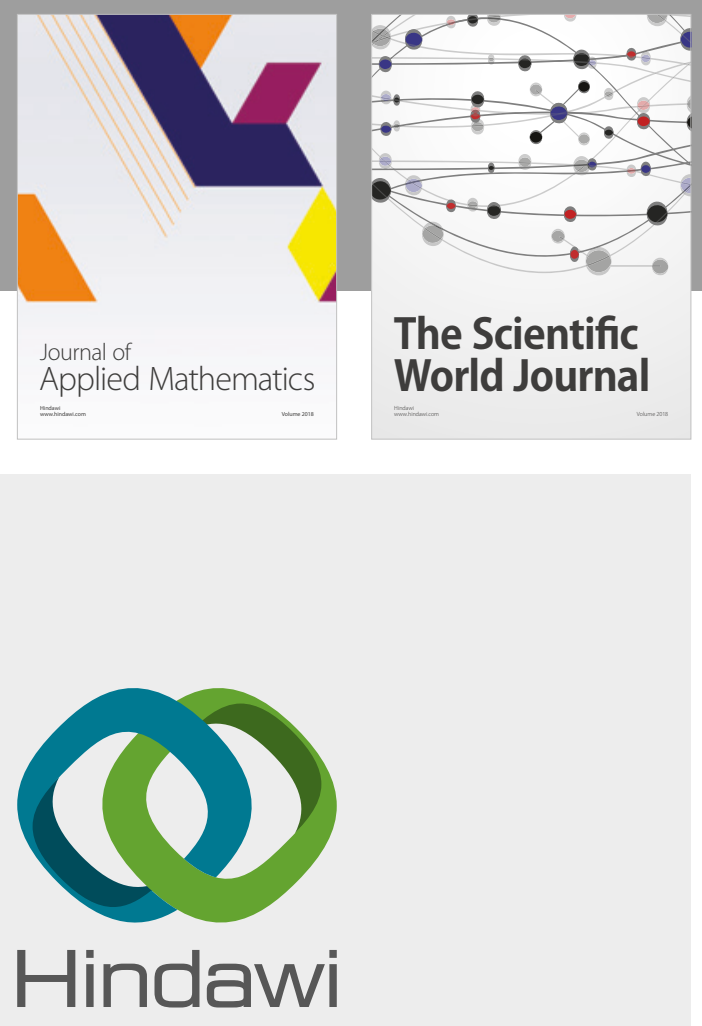

Submit your manuscripts at

www.hindawi.com

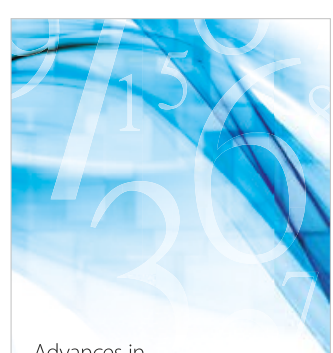

Advances in
Numerical Analysis
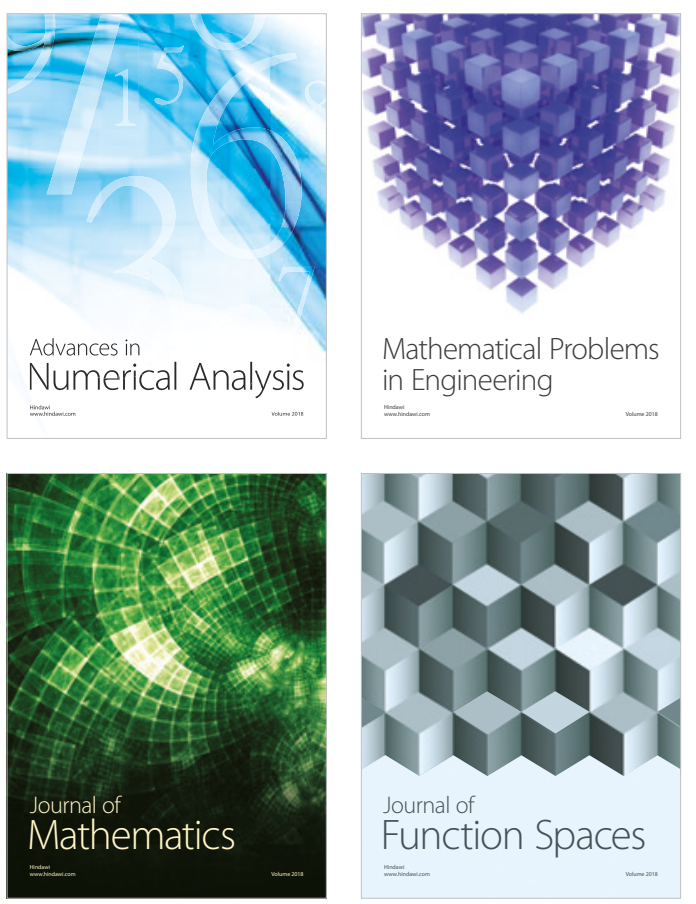

Mathematical Problems in Engineering

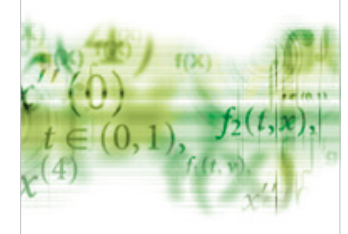

International Journal of

Differential Equations

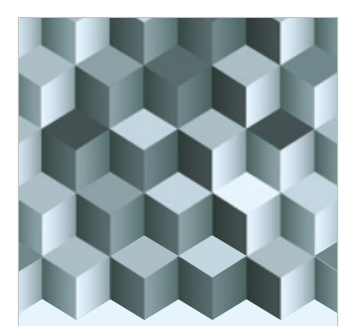

Journal of

Function Spaces

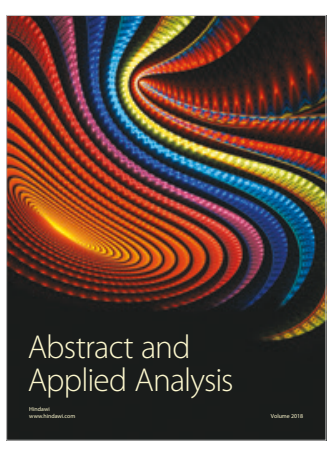

The Scientific

World Journal

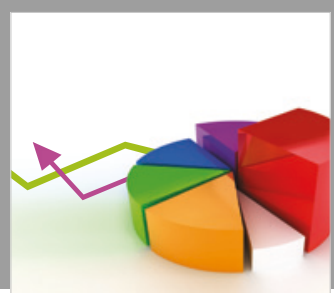

Journal of

Probability and Statistics
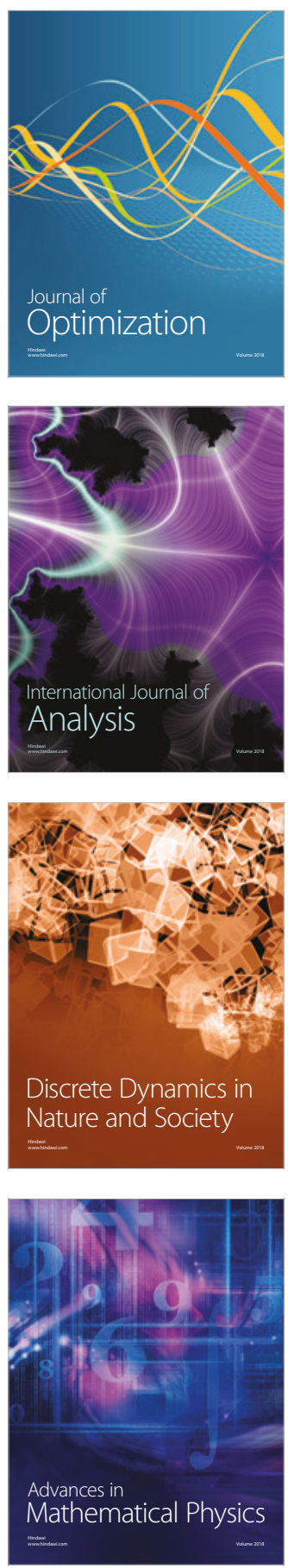